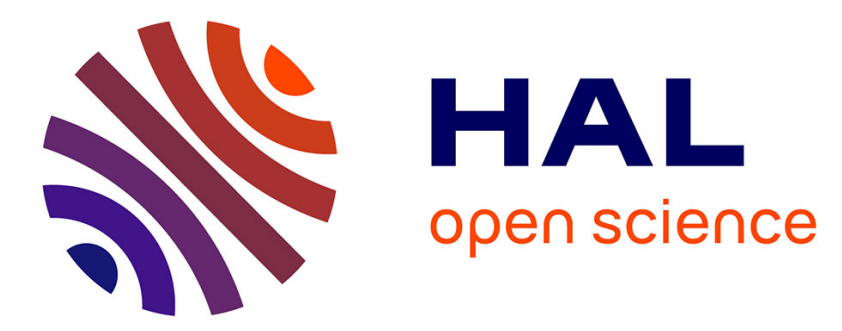

\title{
Influence of the Magnetic Subdivision on the High Frequency Wave Propagation in Polycristalline Soft Ferrites
}

\author{
K. Berthou-Pichavant, F. Liorzou, Ph. Gelin, M. Le Floch
}

\section{- To cite this version:}

K. Berthou-Pichavant, F. Liorzou, Ph. Gelin, M. Le Floch. Influence of the Magnetic Subdivision on the High Frequency Wave Propagation in Polycristalline Soft Ferrites. Journal de Physique IV Proceedings, 1997, 07 (C1), pp.C1-441-C1-442. 10.1051/jp4:19971178 . jpa-00254826

\section{HAL Id: jpa-00254826 https://hal.science/jpa-00254826}

Submitted on 1 Jan 1997

HAL is a multi-disciplinary open access archive for the deposit and dissemination of scientific research documents, whether they are published or not. The documents may come from teaching and research institutions in France or abroad, or from public or private research centers.
L'archive ouverte pluridisciplinaire HAL, est destinée au dépôt et à la diffusion de documents scientifiques de niveau recherche, publiés ou non, émanant des établissements d'enseignement et de recherche français ou étrangers, des laboratoires publics ou privés. 


\title{
Influence of the Magnetic Subdivision on the High Frequency Wave Propagation in Polycristalline Soft Ferrites
}

\author{
K. Berthou-Pichavant, F. Liorzou*, Ph. Gelin and M. Le Floch* \\ LEST/ Télécom Bretagne, Technopôle de Brest Iroise, BP. 832, 29285 Brest cedex, France \\ * LEST/UFR Sciences, 6 avenue Le Gorgeu, 29285 Brest cedex, France
}

\begin{abstract}
In order to examine guided wave propagation in unsaturated ferrites, it seems reasonable to consider an alternation of layers with antiparallel magnetization in a rectangular waveguide. This approach is coherent with Schlömann's model which considers partially magnetized ferrites as an alternation of antiparallel coaxial cylinders. The state of magnetization will be changed by modifying the thickness of antiparallel layers. Globus model, that represents the partially magnetized material as a single grain subdivided into two domains with antiparallel magnetization, seems to be well adapted to express the "magnetization-field" relation.
\end{abstract}

\section{INTRODUCTION}

Due to their gyromagnetic properties, ferrites are being more and more used in microwave devices. Their utilization requires the knowledge of the permeability tensor that is usually established by empirical or physical models. Statistical or mixing laws have been used to modelize those heteregeneous materials. However, all these approaches do not really take the domain subdivision into account.

Taking inspiration from Schlömann's modelization, this paper presents the use of mode matching technique to simulate the wave propagation in non saturated ferrites. The magnetic state is determined by an extension of Globus model.

\section{WAVE PROPAGATION IN UNSATURATED FERRITES: USE OF GLOBUS MODEL}

\subsection{Globus model}

The calculation of the magnetization as a function of the field is the principal problem to establish a permeability tensor model. The Schlömann's subdivision into antiparallel domains allows to consider the use of Globus model.

In fact, Globus model postulates that the general magnetic behaviour of a polycristailine soft ferrite is quite similar to that of a single grain subdivided into two antisymetrical domains. These domains are separated by a $180^{\circ}$ domain wall, whose position is changing in the grain with the magnetic field. A recent extension of Globus model [1] gives a good description of the magnetization curves and hysteresis loops. We propose to apply the results to the simulation of the wave propagation in an alternance of antiparallel layers in a rectangular waveguide, as presented below.

\subsection{Wave propagation in heteregeneous anisotropic magnetic materials}

In this section we examine the wave propagation in a non saturated ferrite that fills a rectangular waveguide. As a result of Schlömann's model [2], and according to Globus extension [1], the unsaturated ferrite can be assimilated to an alternation of respectively "up" magnetized layers (with a magnetization parallel to the external field) and "down" magnetized layers (with a magnetization antiparallel to the external field) layers. One considers a periodic alternation of layers in the guide. The motif is constituted by two thin layers assimilated to the Globus grain. Consequently, the volume of the layers is determined by the Globus model extension and describes the partially magnetized state. The approach with a lot of layers has the advantage to take interactions between the different grains into account. 
Excitation at the guide input is a TE10 mode. Thanks to the mode matching technique and the Floquet's theorem (that takes th periodicity into account), it is possible to determine the dispersion diagram of the structure for different magnetization states [3].
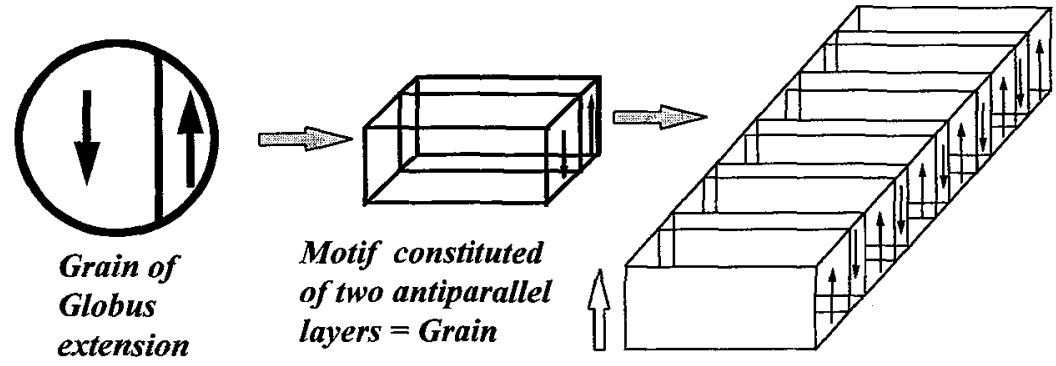

Hext

Figure 1: Common use of Globus modelization and Schlömann's representation to simulate wave propagation in non saturated ferrites

The dispersion diagrams for two different magnetization states $(\mathrm{M} / \mathrm{Ms}=0$ and $\mathrm{M} / \mathrm{Ms}=0.9)$ are presented below (figure $2 \mathrm{a}$ ). $\mathrm{Ms}$ the saturation magnetization and $M$ the partial magnetization. In the case of a totally demagnetized state (the motif is the constituted of two layers with the same volume) it is possible to compare the results with Schlomann's model as shown on figu $2 \mathrm{~b}$. We notice that curves have the same shape, but are frequency shifted. This could be explained by the different geometry of $t$ two modelizations. Yet this check constitutes a validity for the presented method.

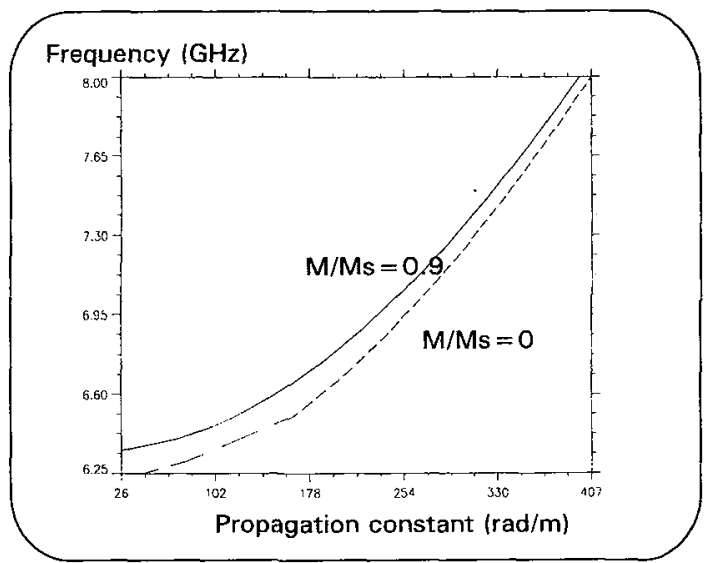

a)

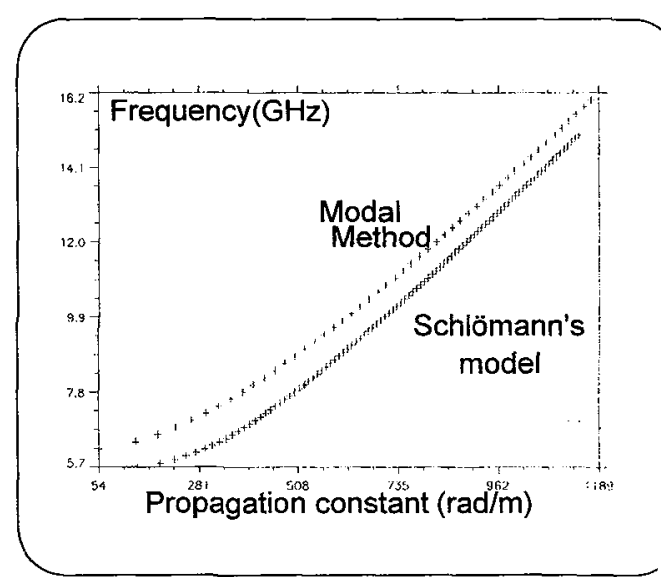

b)

Figure 2: Dispersion diagrams in non saturated ferrites

\section{CONCLUSION}

The combination of Globus model and TE10 wave propagation in a magnetic periodic medium leads to the determination dispersion diagrams in partially magnetized ferrites. Examination of the inverse problem makes it possible to determine components of the permeability tensor. The presented configuration considers only domains with a rectangular geometry, but $i$ possible to introduce more realistic structures. The treatment consists in using a spatial discretization method, such as FDTD. T method is based on discretization of Maxwell's and magnetic moment equations, and would be particularly well adapted describe the physical subdivision in magnetics domains of the partially magnetization state [3].

\section{Références}

[1] Liorzou F., Konn A.M., Le Floc'h M., "A common expression for both direct and inverse magnetization curves in ! ferrites", JMMM, 140-144, (1995), 1887

[2] Schlömann, "Microwave Behavior of partially magnetized ferrites", Journal of Applied Physics, vol. 41, $n^{\circ} 1,(1970)$

[3] Berthou-Pichavant K., and Gelin Ph., "Wave propagation in non saturated ferrites: Use in modelling", ISEM, (1995), F35 